\title{
Distribución potencial del tapir centroamericano en la península de Yucatán
}

\section{Natalia Carrillo ${ }^{1^{*}}$, Holger Weissenberger ${ }^{2}$ y Rafael Reyna-Hurtado ${ }^{3}$}

\begin{abstract}
${ }^{1}$ Maestría en Ciencias en Recursos Naturales y Desarrollo Rural, Manejo y Conservación de los Recursos Naturales, El Colegio de la Frontera Sur. Ave. Centenario Km 5.5 Carretera Calderitas, Chetumal 77900. Quintana Roo, México. E mail: ncarrillo@ecosur.edu. $m x(N C)$.

${ }^{2}$ Dirección Académica, Laboratorio de Análisis de Información Geográfica y Estadística, El Colegio de La Frontera Sur. Ave. Centenario Km 5.5 Carretera Calderitas, Chetumal 77900. Quintana Roo, México. E mail: holgerweissen@ecosur.mx (HW).

${ }^{3}$ Ecología para la Conservación de la Fauna Silvestre, Departamento de Conservación de la Biodiversidad, El Colegio de la Frontera Sur. Av. Rancho Polígono 2A, Parque Industrial, Lerma, Campeche 24500, México. Email: rreyna@ecosur.mx (RRH).

* Autor de correspondencia
\end{abstract}

Introduction: The habitat is used differentially by different species, depending on their feeding and breeding habits, space requirements and tolerance to areas with lower quality conditions. The way we understand the requirements for each species and individual will be different according to the spatial scale of habitat being studied, therefore habitat selection must be analyzed at different spatial scales. In a wide scale (biogeographic) the establishments of a species are mainly influenced by types of climte, vegetation and altitude. At a local scale (i.e. in a $10 \mathrm{~km}^{2}$ patch) the variables that influence the presence of the species could be food availability, predation risk and competition. Knowing the spatial distribution of an organism at regional and biogeographic scales and the factors that influence them, will allow to propose conservation areas targeted for a species or population, since its geographic variation allows us to identify areas with higher value for ecological conservation. The aim of this study was to evaluate the potential distribution of Baird's tapir (Tapirus bairdii) in the Yucatan peninsula, México and determine the climatic variables that influence such distribution.

Methods: We performed a species distribution modeling approach using a Maximum Entropy analysis (Maxent; Phillips et al. 2006) that use environmental variables to predict the potential distribution of an organism in a given geographic area given presence records. To obtain the tapir occurrence records a search was performed on academic and governmental information sources (Appendix I). Only data obtained in the Yucatan peninsula over the past 10 years were selected. For the model generation we used the bio-climatic variables obtained from WorldClim, which provides global climate layers with information derived from monthly values of temperature and precipitation.

Results: According to the influence of climate variables, the tapirs in the Yucatan peninsula occur in places with high rainfall and when the temperatures are not high (Figure 3). The answer to these variables confirms the need for this mammal to inhabit areas where sufficient water is ensured for his maintenance. Additionally, potential distribution areas were identified where studies are required to confirm the presence of the species and the possibility to integrate them into conservation plans.

Discussion and conclusions: The potential distribution model suggests areas where tapirs can be distributed but there are no presence records and where records are only for sightings, as is the central part of the state of Campeche, close to forests of the Biosphere Reserve of Sian Ka'an, and the coastal strip Majahual-Xcalak and around Lake Bacalar in the state of Quintana Roo. Studies on these sites are recommended to check for the presences of tapirs. The potential distribution model is a useful tool to propose conservation and management plans in areas that do not have any protection, and to know the climate variables suitable for the distribution of the species that may be applicable at the time of proposing biological corridors. It is also important to be aware of change in land use in areas where yet the tapir is distributed to carry out management plans and conservation of these areas.

Key words: connectivity; land change use; natural protected area; potential distribution. 
Introducción: El hábitat es usado de forma diferente por distintas especies, dependiendo de su alimentación, hábitos reproductivos, requerimientos de espacio y tolerancia a áreas con condiciones de menor calidad. La forma en que entendemos los requerimientos de cada especie e individuo será diferente de acuerdo con la escala espacial del hábitat que se estudie; por lo tanto, la selección de hábitat debe ser analizada a diferentes escalas espaciales. A una escala amplia (biogeográfica), el establecimiento de una especie se verá influido por los tipos de clima, vegetación y altitud. A una escala local (i. e. en un parche $10 \mathrm{~km}^{2}$ ), las variables que influyen sobre la presencia de las especies pueden ser la disponibilidad de alimento, el riesgo de depredación y la competencia. Conocer la distribución espacial de un organismo a escalas regionales y biogeográficas, así como los factores que influyen sobre ésta, resulta de gran utilidad para proponer áreas de conservación enfocadas en una especie o población, ya que su variación geográfica nos permite señalar las áreas con mayor valor ecológico para su conservación. El objetivo de este estudio fue evaluar la distribución potencial del tapir centroamericano (Tapirus bairdii) en la península de Yucatán, y determinar las variables climáticas que influyen en dicha distribución.

Métodos: Para ello, se desarrolló un modelo de distribución de especies utilizando el análisis de Máxima Entropía (Maxent; Phillips et al. 2006) que usa variables ambientales para predecir la distribución de un organismo en un área geográfica dada con base en los registros de presencia de la especie. Para obtener los registros de tapir, se buscaron datos de presencia en diferentes fuentes de información, tanto académicas como gubernamentales (Apéndice I). Únicamente se seleccionaron los datos obtenidos en la península de Yucatán durante los últimos 10 años. En cuanto a la parte climática, se utilizaron las variables bioclimáticas obtenidas de WorldClim, las cuales proporcionan capas con información climática global derivadas de valores de temperatura y precipitación mensuales.

Resultados: De acuerdo con la influencia de las variables climáticas, la mayor probabilidad de encontrar tapires en la península de Yucatán se da en lugares donde llueve mucho y las temperaturas no son muy altas (Figura 3). La respuesta a dichas variables confirma la necesidad de este mamífero de habitar en zonas donde se asegure una cantidad suficiente de agua para sobrevivir. Adicionalmente, se identifican zonas de distribución potencial por las características climáticas idóneas en las que el tapir puede distribuirse. Sin embargo, se requieren estudios para descartar o confirmar la presencia de la especie y contemplar la posibilidad de integrar estas zonas a planes de conservación, como áreas donde los registros correspondan únicamente a avistamientos y atropellamientos, y donde el modelo confirme una fuerte probabilidad de presencia de tapires.

Discusión y conclusiones: El modelado de distribución potencial de las especies es una herramienta útil para proponer planes de conservación y manejo en zonas que aún no cuentan con algún nivel de protección. Ejemplos de lo anterior son la parte central del estado de Campeche, los bosques cercanos a la Reserva de la Biosfera de Sian Kaan y la franja costera Majahual-Xcalak, así como los alrededores de la laguna de Bacalar, en el estado de Quintana Roo. Conocer las variables climáticas aptas para la distribución de la especie puede ser de gran utilidad en el momento de proponer corredores biológicos. Asimismo, es importante conocer el cambio de uso de suelo en las áreas donde aún se distribuye el tapir, a fin de llevar a cabo planes de manejo y conservación de estas zonas.

Palabras clave: área natural protegida; cambio de uso de suelo; conectividad; distribución potencial.

\section{Introducción}

La extensión y resolución espacial y temporal de un estudio pueden afectar nuestra forma de observar los fenómenos en un sistema y las inferencias que se extraen de ellos (López de Casenave et al. 2007). La importancia de la escala radica en que cada proceso ecológico ocurre a escalas espaciales y temporales muy diferentes (Levin 1992; Turner et al. 2001). Los patrones de distribución de los organismos suceden en respuesta a las variaciones físicas del ambiente; por ejemplo, en hábitats terrestres, estos patrones son determinados por el clima, los tipos de suelo y la fisiología del animal (Brown y Lomolino 1998). La selección de hábitat, por lo tanto, debe ser analizada a diferentes escalas espaciales, ya que la forma en que entendemos los requerimientos de cada especie e individuo será diferente de acuerdo con la escala espacial que se estudie (Saab 1999; Goulart et al. 2009; Chavez 2010). A una escala amplia (biogeográfica), el establecimiento de una especie se verá influido principalmente por los tipos de clima, vegetación y altitud, entre otros factores. A una escala local (i. e. en un parche de $10 \mathrm{~km}^{2}$ ), las variables que influyan pueden ser la disponibilidad de alimento, el riesgo de depredación y las interacciones competitivas (Pedlar et al. 1997; Pérez-Solano 2011).

Es fundamental, entonces, conocer cuáles son los requerimientos ambientales, biológicos y ecológicos de los organismos, y a qué escala ocurren para así interpretar cómo reaccionan a los 
cambios ambientales y a la reducción de los ambientes naturales, en particular, las especies en peligro de extinción. Lo anterior nos permitirá conocer mejor el hábitat al que se encuentran asociadas dichas especies y así desarrollar estrategias de manejo y conservación (Foerster y Vaughn 2002; Hernández et al. 2006; de Villa Meza 2006; Goulart et al. 2009).

En el ámbito global, la Unión Internacional para la Conservación de la Naturaleza considera al tapir centroamericano (Tapirus bairdii) en peligro de extinción (UICN 2013; Castellanos et al. 2008). En México, la Secretaria de Medio Ambiente y Recursos Naturales lo considera también en peligro de extinción, así como especie prioritaria de conservación (NOM-059-SEMARNAT-2010). Es el mamífero terrestre más grande del neotrópico, una de las cuatro especies que conforman la familia Tapiridae y una de las tres que se encuentran en el continente americano (Tobler 2006; Mendoza y Carbajal 2011). Su distribución geográfica incluye el área comprendida desde el sureste de México hasta el norte de Colombia (Reid 1997; Lawton 2000; Kappelle y Brown 2001; Naranjo 2002). Sin embargo, en los últimos 30 años, las poblaciones de tapir Centroamericano se han reducido en $50 \%$ en toda su área de distribución (Castellanos et al. 2008). En México, su distribución actual abarca solamente alrededor de $45 \%$ de lo que era su distribución histórica (Nolasco et al. 2007). Dicha reducción en las poblaciones se debe principalmente a la deforestación y a la fragmentación de su hábitat, a la cacería y a las muertes producidas en encuentros incidentales con humanos (Robinson y Redford 1994; Naranjo 2009).

El sureste del país constituye una región crítica para conservar el tapir centroamericano, en virtud de la extensión de hábitats adecuados (selvas y humedales) que aún mantiene, así como del tamaño de las poblaciones que concentra: según estimaciones, cerca de $50 \%$ del total de la población global (Naranjo 2009; Mendoza y Carbajal 2011). Si bien existe consenso en cuanto al papel crítico que desempeñan las grandes reservas del sureste del país (Montes Azules, La Sepultura, El Triunfo, Sian Ka'an y Calakmul), los estudios, en su mayoría, se han llevado a cabo en las áreas naturales protegidas del estado de Chiapas. Existe muy poca información sobre la importancia de las reservas en la península de Yucatán; tampoco se han tomado en cuenta los grandes parches de hábitat conservado que todavía permanecen sin protección, ni los ejidos con ampliaciones forestales para mantener las poblaciones de esta especie (Mendoza y Carbajal 2011).

Esta investigación tuvo como objetivo evaluar la distribución potencial del tapir centroamericano en la península de Yucatán, en el sur de México. Se basa en el modelado de distribución de especies y utiliza un análisis de Máxima Entropía (Maxent; Phillips et al. 2006). Fueron identificadas áreas importantes para la especie y su conservación, así como las variables ambientales relevantes que influyen en su distribución a una escala biogeográfica.

\section{Materiales y métodos}

Área de estudio. La península de Yucatán cubre un territorio de 138,000 km² (CRUPY 2007) y está integrada por los estados mexicanos de Yucatán, Quintana Roo y Campeche. No obstante, estudios geofísicos de Lugo-Hubp y colaboradores (1992) indican que junto a Belice y el Petén, en Guatemala, se conforma una gran cuenca. La península presenta un gradiente de humedad del noroeste hacia el sureste (Gunn y Adams 1981; Folan et al. 1983) con precipitaciones que varían de $600 \mathrm{~mm}$ en el norte a 1,400 mm en el sur. En la península hay una temporada de lluvias, principalmente entre junio y octubre, y una temporada de secas con mayor intensidad entre febrero y mayo (Vester et al. 2007; Guerra 2010). Uno de los rasgos más peculiares de la península es la ausencia de prominencias morfológicas de importancia, de tal forma que $90 \%$ de su superficie se encuentra a menos de 200 metros de elevación (Kauffer y Villanueva 2011).

Registros de presencia. Para aplicar el modelo, se elaboró una base de datos con los registros georreferenciados de tapir centroamericano en la península de Yucatán durante los últimos 10 
años (Apéndice II). A fin de obtener estos registros, se buscaron datos de la presencia del tapir; para ello, fueron consultadas las siguientes fuentes:

1) Literatura especializada y literatura gris (tesis y publicaciones en talleres o congresos) en los que se reporta a la especie. Se extrajeron bases de datos de diversas investigaciones publicadas y no publicadas que han trabajado con el tapir centroamericano en la región. Estas últimas fueron solicitadas y proporcionadas por los investigadores y estudiantes que las elaboraron.

2) Investigadores y personas especializadas en el tema y en la región, a quienes se les solicitó datos de registros individuales de tapir aún no publicados. En su mayoría, fueron datos de avistamientos y atropellamientos que poseen los investigadores y los pobladores, pero que no se han registrado en ninguna publicación ni trabajo científico. No hay planes a corto plazo para publicarlos.

3) Direcciones de áreas naturales protegidas en la península de Yucatán, a las que se les solicitó datos de avistamientos y rastros de tapir. Cabe mencionar la Reserva de la Biosfera de Sian Ka'an, el parque natural Xcalak, el área de protección de flora y fauna Balam Ka'ax y el área de protección de flora y fauna Yum Balam, en Quintana Roo. Particularmente, en las reservas de Yucatán no hubo posibilidad de contactar direcciones ni se encontraron personas enteradas de registros confiables de tapir en la región. Tampoco se obtuvo conocimiento de registros o avistamientos en la reserva de los Petenes, en Campeche.

4) Colecciones científicas en la base de datos de la Comisión Nacional para el Conocimiento y Uso de la Biodiversidad (CONABIO) y en la colección de El Colegio de la Frontera Sur, unidad Chetumal.

Información climática. Como variables ambientales, se utilizó 11 capas bioclimáticas disponibles en WorldClim (Hijmans et al. 2005). Se seleccionaron estas variables (Tabla 1) por tener un mayor aporte en la generación del modelo de acuerdo con la ejecución, en repetidas ocasiones, del modelo con la aplicación de todas las variables ambientales disponibles (19 variables) y su posterior discriminación y selección (Phillips et al. 2006). Las variables bioclimáticas son derivadas de valores de temperatura y precipitación mensuales, con objeto de generar variables con mayor aplicación biológica. Dichas variables pueden presentarse como tendencias anuales (e. g. media de temperatura y precipitación anual), estacionales (e. g. rango anual de temperatura y precipitación), y factores ambientales limitantes o extremos (e. g. temperatura o precipitación del mes más frío o más cálido). Se trabajó en todas las variables a una resolución de pixel de 1 km².

Con la finalidad de estimar cuáles son las variables más importantes en el modelo, se realizó un análisis de Jackknife, que crea un conjunto de modelos en los cuales excluye una variable en

Tabla 1. Capas climáticas utilizadas para elaborar el modelo de distribución potencial del tapir centroamericano en la península de Yucatán, México. Fueron ordenadas de acuerdo con el aporte en la generación del modelo.

\begin{tabular}{ll}
\multicolumn{1}{c}{ Clave } & \multicolumn{1}{c}{ Descripción de la variable } \\
\hline Bio $\mathbf{1 3}$ & Precipitación del mes más lluvioso $(\mathrm{mm})$ \\
Bio $\mathbf{2}$ & Media del intervalo diurno (media del mes (max-temp-min temp)) \\
Bio 8 & Temperatura media del trimestre más lluvioso $\left({ }^{\circ} \mathrm{C}\right)$ \\
Bio $\mathbf{1 9}$ & Precipitación del trimestre más frio $(\mathrm{mm})$ \\
Bio $\mathbf{1 5}$ & Coeficiente de variación de las precipitaciones estacionales \\
Bio $\mathbf{9}$ & Temperatura media del trimestre más seco $\left({ }^{\circ} \mathrm{C}\right)$ \\
Bio 6 & Temperatura mínima del mes más frio $\left({ }^{\circ} \mathrm{C}\right)$ \\
Bio $\mathbf{1 1}$ & Temperatura media del trimestre más húmedo $\left({ }^{\circ} \mathrm{C}\right)$ \\
Bio $\mathbf{5}$ & Temperatura máxima del mes más caliente $\left({ }^{\circ} \mathrm{C}\right)$ \\
Bio $\mathbf{1 6}$ & Precipitación del trimestre más lluvioso $(\mathrm{mm})$ \\
Bio $\mathbf{1 2}$ & Precipitación anual $(\mathrm{mm})$ \\
\hline
\end{tabular}


cada iteración y crea un modelo con las variables restantes; asimismo, crea un modelo usando cada variable sola, de manera aislada, y para tener un comparativo entre ambos modelos, se crea, adicionalmente, otro modelo con todas las variables juntas.

Modelado de distribución potencial. Para modelar la distribución potencial del tapir, se realizó un modelado de distribución de especies con el análisis de Máxima Entropía (Maxent; Phillips et al. 2006) y con base en los registros espaciales de presencia de la especie y de las variables ambientales limitadas al área de estudio. Este modelo proporciona conocimiento sobre los requerimientos ambientales de las especies y los factores que influyen en su adaptación al ambiente (e.g. el tipo de clima, la vegetación, la altura o el relieve). De esta manera, se logra inferir qué características del ambiente en un área determinada serán las más idóneas para la sobrevivencia del tapir (Phillips et al. 2006). Con el propósito de evaluar la ejecución del modelo, se eligió la opción "porcentaje de prueba al azar", con la que se separa un porcentaje de datos para crear un modelo paralelo de validación. Para ello, se eligió $75 \%$ de los datos como registros de entrenamiento (eliminados los datos duplicados) y $25 \%$ como datos de evaluación seleccionados al azar. Maxent, además, proporciona la opción de aplicar el mismo modelo en múltiples ocasiones para promediar los resultados; en consecuencia, se realizaron 15 réplicas que aportaron información sobre la cantidad de variabilidad del modelo (Young et al. 2011). Maxent elabora un mapa en formato raster con valores de 0 a 1, que representa la probabilidad de encontrar las mejores condiciones para la especie (Young et al. 2011). Los valores umbrales de idoneidad se basan en una validación estadística que el modelo realiza por medio de una prueba binomial. En este caso, para conocer todas las posibilidades de distribución de los tapires, se eligió el "umbral mínimo logístico de presencia".

Para probar el rendimiento del modelo y la capacidad discriminatoria, se utilizó la información de la curva ROC (Receiver Operating Characteristic) y el valor AUC de evaluación (Area Under the Curve). Un valor de AUC cercano a 0.5 indica que la ejecución del modelo no es mejor que el azar, mientras que valores cercanos a 1 indican un mejor desempeño del algoritmo (Phillips et al. 2006).

\section{Resultados}

En total, se obtuvo 265 registros de tapir centroamericano para ejecutar el modelo (Apéndice II). Se obtuvo un valor de área bajo la curva (AUC) de 0.939 para los datos de entrenamiento, y 0.913 para los datos de validación. Lo anterior indica buen desempeño y capacidad discriminatoria del modelo. Las principales variables ambientales para predecir la presencia del tapir centroamericano son 1) La temperatura media del trimestre más lluvioso. 2) La precipitación del mes más lluvioso. 3) El intervalo de temperatura media diurna. 4) La temperatura media del trimestre más seco. 5) La precipitación del trimestre más lluvioso. 6) La temperatura mínima del mes más frío. Estas variables contribuyen en casi $89 \%$ para predecir la presencia de la especie (Tabla 2).

De acuerdo con la prueba de Jackknife, al desarrollar un modelo con cada variable de manera aislada, la que contribuye con una mayor ganancia en la generación del modelo por sí sola es la temperatura media del trimestre más lluvioso. Las variables cinco (precipitación del mes más lluvioso) y cuatro (temperatura media del trimestre más seco) resultaron ser irremplazables, ya que al ser eliminadas del modelo contribuyen a que el mayor ajuste del modelo se pierda (Figura 1). 
Tabla 2. Estimado de contribución de las variables ambientales para elaborar el modelo de distribución potencial del tapir centroamericano en la Península de Yucatán, México.

\begin{tabular}{lc}
\multicolumn{1}{c}{ Variable } & \% de contribución \\
\hline Temperatura media del trimestre más lluvioso $\left({ }^{\circ} \mathrm{C}\right)($ bio_8) & $23.10 \%$ \\
Precipitación del mes más Iluvioso $(\mathrm{mm})($ bio_13) & $18.10 \%$ \\
Media del intervalo diurno (media del mes(max-temp-min temp)) (bio_2) & $17.00 \%$ \\
Temperatura media del trimestre más seco $\left({ }^{\circ} \mathrm{C}\right)($ bio_9) & $11.90 \%$ \\
Precipitación del trimestre más lluvioso $(\mathrm{mm})($ bio_16) & $10.70 \%$ \\
Temperatura mínima del mes más frío $\left({ }^{\circ} \mathrm{C}\right)($ bio_6) & $7.70 \%$ \\
\hline
\end{tabular}

Mapa de distribución potencial. En este caso, el "umbral mínimo logístico de presencia" indica que las condiciones más adecuadas para verificar la presencia del tapir se encuentran por arriba del umbral 0.37, y las menos adecuadas, por debajo de él (es decir, 0.37 indica las zonas con pocas condiciones idóneas para el tapir; Figura 2). Dos zonas de distribución potencial sugeridas, y donde aún no se cuenta con registros confiables de la especie, son el centro del estado de Campeche (entre los poblados de Yohaltun, Pich y Dzibalchen) y la parte este del estado de Quintana Roo (entre Santa Rosa, Felipe Carrillo Puerto, Petcacab y Limones). La franja costera Majahual-Xcalak es otra área predicha, la cual se delimita como una zona con condiciones adecuadas para la especie.

\section{Discusión}

Los tapires habitan preferentemente en áreas boscosas extensas, con poca perturbación humana y presencia permanente de agua (March y Naranjo 2005). Sin embargo, en la búsqueda de recursos, pueden llegar a utilizar varios tipos de hábitat a través del paisaje (Foerster y Vaughan 2002; Naranjo 2009; Lira-Torres y BrionesSalas 2012; Carrillo et al. 20135). En México, el tapir centroamericano se distribuye en bosques tropicales y bosques de montaña, en altitudes que van desde los 0 hasta

Figura 1. Importancia de cada variable climática en el modelo de distribución potencial, de acuerdo con la prueba de Jackknife. La línea roja representa la ganancia del modelo utilizando todas las variables; las líneas azules muestran la ganancia del modelo utilizando cada variable de manera aislada, y las líneas turquesas representan la ganancia del modelo cuando se excluye cada variable.

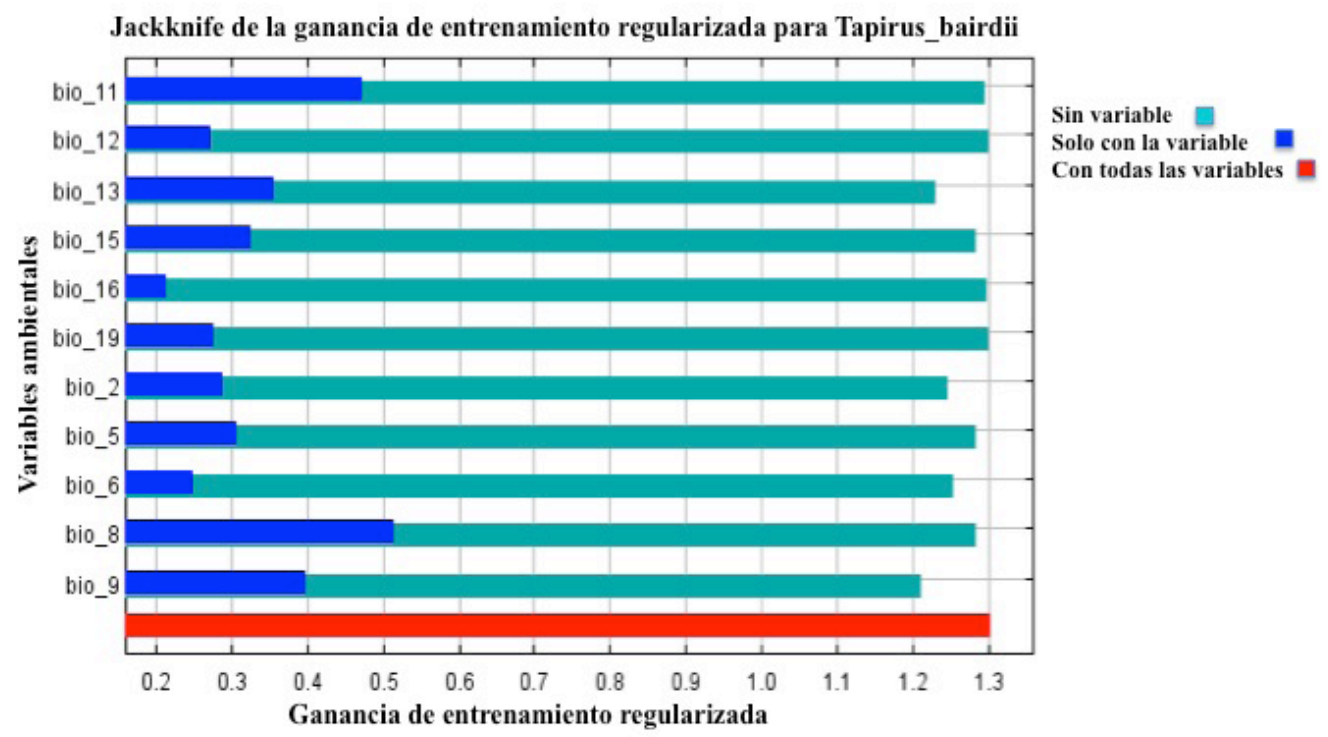


los 2,000 m (Naranjo 2009); abarcan gran variedad de climas, desde los costeros y con poca precipitación hasta el bosque nuboso. En Costa Rica, se ha reportado la presencia de tapir centroamericano arriba de los 3,600 m (Naranjo y Vaughan 2000; F. Carbonell, comunicación personal, noviembre 2012). Pese a lo anterior, la acelerada pérdida de hábitat provoca que los tapires se vean relegados a las pocas áreas naturales protegidas; por lo tanto, es importante delimitar sus preferencias climáticas, para, junto con el conocimiento que se ha generado acerca de sus preferencias de hábitat, se pueda inferir con más certeza la gama completa de requerimientos ecológicos en el intento de someter nuevas áreas de distribución potencial a la protección legal.

El presente estudio concuerda con los datos conocidos acerca de los requerimientos ambientales de los tapires. Es posible inferir que la influencia de la variable "precipitación del mes más lluvioso" en la presencia del tapir puede deberse a que una mayor cantidad de lluvia asegura mayor producción vegetal y mayor disponibilidad de agua. La respuesta a dicha variable confirma, además, la necesidad de este mamífero de habitar en zonas donde se asegure una cantidad suficiente de agua para su mantenimiento. Las variables "temperatura del trimestre más lluvioso" (julio-octubre: CONAGUA) y "precipitación del mes más lluvioso" (agosto-octubre; CONAGUA; Mardero et al. 2012) pueden estar correlacionadas, de manera que la mayor probabilidad de encontrar tapires en la península de Yucatán se da en lugares donde llueve mucho y las temperaturas no son muy altas (Figura 3). Similares resultados encuentra Nolasco (2009), quien realizó un modelo para conocer la distribución actual e histórica del tapir en México y señala que la variable ambiental que obtuvo un mayor porcentaje en la determinación de la presencia del tapir fue la precipitación anual.

Figura 2. Mapa de distribución potencial de Tapirus bairdii en la península de Yucatán. El color rojo representa las áreas con las mejores condiciones para la presencia de la especie; el verde turquesa, las zonas con condiciones medias, y el azul, las zonas de baja o nulas condiciones aptas para la especie. Los puntos negros representan los "datos de entrenamiento" y los amarillos los "datos de validación".

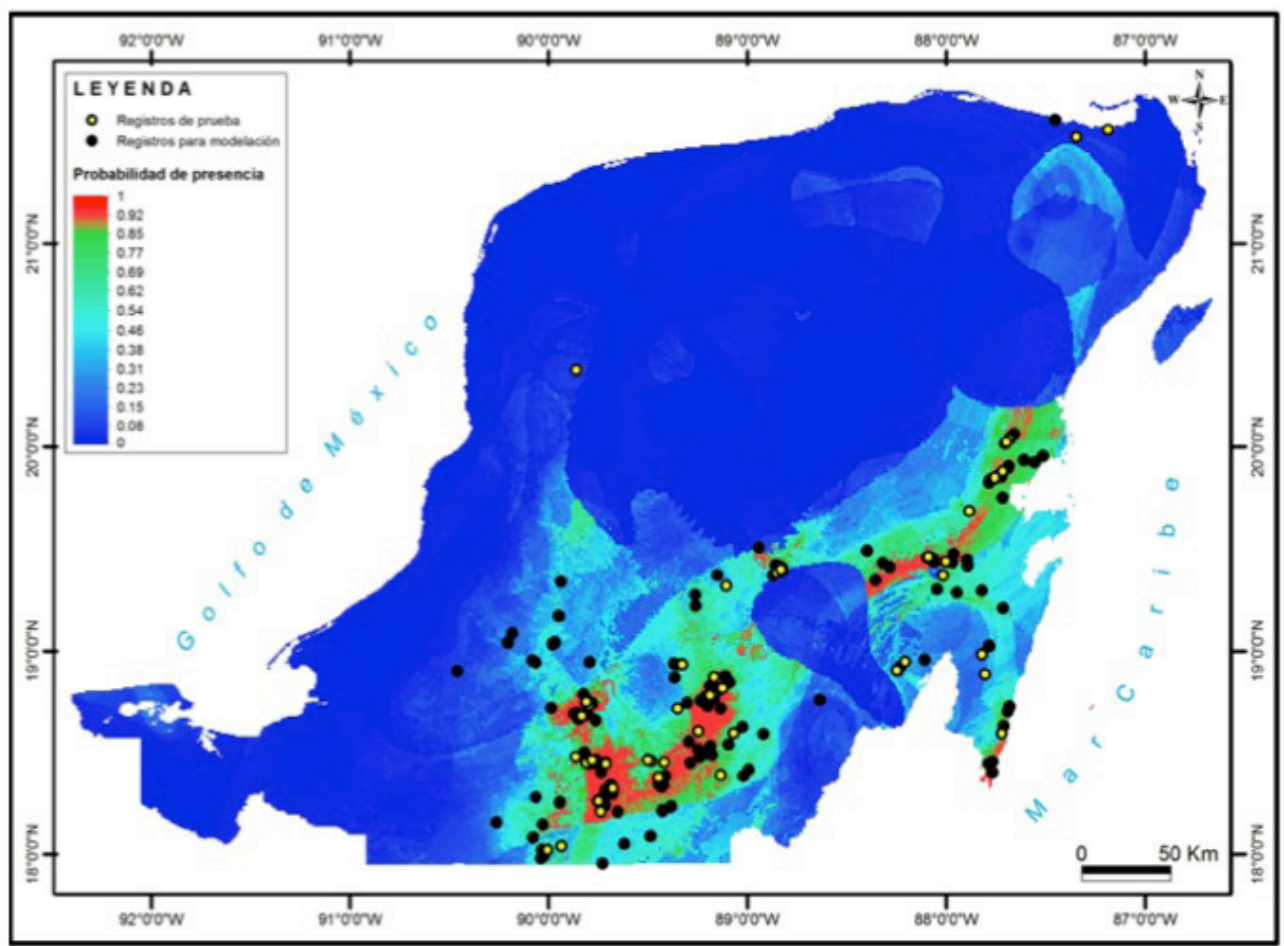


La variable "temperatura del trimestre más seco del año" indica que el tapir se distribuye en zonas cuya temperatura media se encuentra entre 24 y $27^{\circ} \mathrm{C}$ en los meses más secos (febrero-mayo; Mardero 2012; Figura 3). Cuando la temperatura aumenta o disminuye fuera de este intervalo, desciende la probabilidad de presencia del tapir; por ello puede inferirse que esta especie habita preferentemente en zonas donde, en los periodos más secos del año, las temperaturas no sean muy altas. De acuerdo con el mapa de distribución potencial generado en este estudio, el área norte de la península de Yucatán no cuenta con la presencia del tapir, lo cual puede estar relacionado con el efecto del gradiente de humedad presente en la península, que indica un aumento en la precipitación y una disminución de la temperatura que va del norte al sur (Gunn y Adams 1981; Folan et al. 1983). Lo anterior sugiere que los tapires no se distribuyen en zonas más calientes y con escasa precipitación.

Algunas zonas de distribución potencial sugeridas (centro del estado de Campeche y la parte este del estado de Quintana Roo) indican sitios donde se propone llevar a cabo estudios para verificar la presencia y, en tal caso, la abundancia de tapires. Estos resultados coinciden con los de Nolasco (2009), quien priorizó cuatro áreas de distribución en el país, entre ellas la Reserva de la Biosfera de Calakmul y áreas adyacentes, así como la Reserva de la Biosfera de Sian Ka \an y áreas adyacentes. La franja costera Majahual-Xcalak es otra área predicha, la cual se delimita como una zona con condiciones adecuadas para la especie. Aunque en esta región sí se cuenta con registros de la especie, éstos corresponden solamente a datos sobre animales atropellados o avistamientos realizados por pobladores e investigadores (J. Gómez, comunicación personal 2013). En el mapa de distribución potencial (Figura 2), es posible ubicar zonas con características climáticas aptas para los tapires. Es el caso de la franja al sureste de la ciudad de Chetumal y del cauce del Río Hondo en la frontera con Belice. En esta región, se han avistado tapires, específicamente en la Laguna Milagros, ubicada en el poblado de Huay Pix, a 10 minutos de la ciudad de Chetumal (J. Pérez Flores, comunicación personal, marzo 2013).

Figura 3. Curvas de respuesta de Tapirus bairdii a las variables climáticas predichas por el Modelo de Máxima Entropía en la península de Yucatán. Los datos de temperatura están en unidades de ${ }^{\circ} \mathrm{C} \times 10$ (Hijmans, 2006). A) Probabilidad de presencia de T. bairdii, de acuerdo con la temperatura media del trimestre más lluvioso. B) Probabilidad de presencia de T. bairdii, de acuerdo con la precipitación del mes más lluvioso. C) Probabilidad de presencia de T. bairdii de acuerdo con la temperatura del trimestre más seco.

A

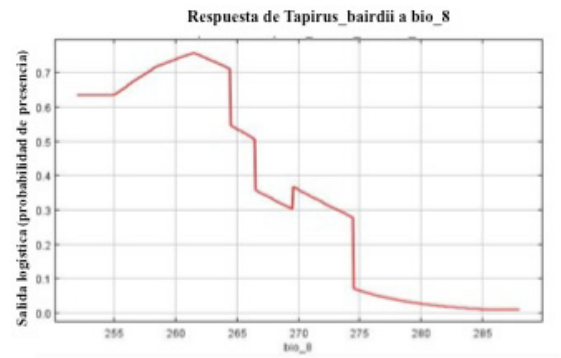

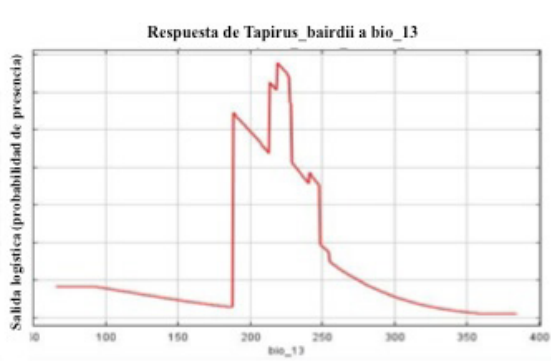

C

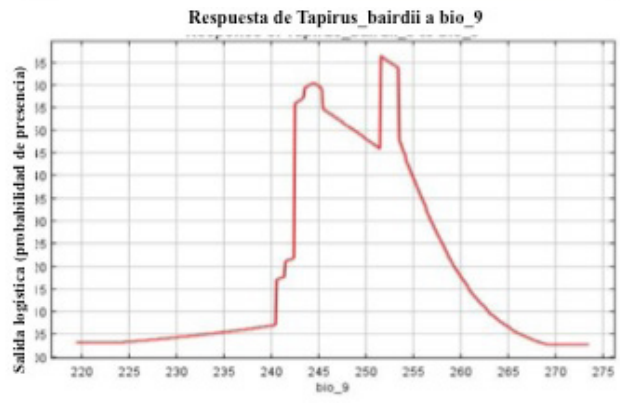


Por otra parte, Vaca et al. (2012) indican que la deforestación en el sureste de México se ha acelerado entre 1999 y 2006. El mapa de distribución potencial muestra áreas aptas para la presencia del tapir; sin embargo, hay que tomar en cuenta la desaparición de selvas para dar paso a zonas de cultivo, lo que afectará sin dudas la presencia de la especie en esas áreas. Es importante conocer el cambio de uso de suelo en las áreas donde aún se distribuye el tapir para llevar a cabo planes de manejo y conservación de esas zonas. Según la carta de vegetación elaborada por Instituto Nacional de Estadística y Geografía (1999), la mayor parte de la península de Yucatán es apta para contar con la presencia de tapir, debido a los bosques tropicales en buen estado de conservación. No obstante, conociendo la influencia de variables climáticas en la presencia de tapir, puede hacerse una delimitación más certera de áreas con probabilidad de presencia.

Si el modelo generado por Maxent en esta investigación, que contempla solo variables climáticas, se complementa y afina con información de uso de suelo y variables de vegetación, puede resultar una herramienta sumamente útil para proponer nuevos sitios de investigación sobre la especie. Igualmente al conocer el estado en el que se encuentran los parches y remanentes de bosque es posible delimitar la presencia de corredores naturales. Si los resultados generados por Maxent son verificados en campo es posible generar programas de manejo y protección de nuevas áreas de distribución de esta especie en peligro de extinción.

\section{Agradecimientos}

Al Consejo Nacional de Ciencia y Tecnología por la beca otorgada (núm. 393532) al primer autor de este articulo para llevar a cabo sus estudios de maestría, así como por la beca otorgada"Becas mixtas 2012" para realizar una estancia de estudio en el extranjero. A todas la personas que colaboraron con datos de registros de tapir para esta investigación. A B. Scmook, S. Calmé, G. O'Farril y E. Nickel, por sus revisiones y comentarios. A. Jasso y N. Arias por la ayuda prestada en campo. Este proyecto fue financiado por el Fondo Sectorial CONACYT-SEMARNAT (clave SEMARNAT-2008-C01-108348).

\section{Literatura citada}

Brown, J. H., y M. V. Lomolino. 1998. Distribution of single species. Pp. 61-93 en Biogeography (Brown, J. H., y M. V. Lomolino, eds.). Segunda edición, Sinauer Associates Incorporation. Massachussetts, EE. UU.

Carrillo, N., R. Reyna-Hurtado, y B. Schmook. 2015. Abundancia relativa y selección de hábitat de Tapirus bairdii en las reservas de Calakmul y Balam kú, Campeche, México. Revista Mexicana de Biodiversidad 86:202-207.

Castellanos, A., C. Foerster, D. J. Lizcano, E. Naranjo, E. Cruz-Aldan, I. Lira-Torres, R. Samudio, S. Matola, J. Schipper, y J. Gonzalez-Maya. 2008. Tapirus bairdii en IUCN 2012. IUCN Red List of Threatened Species. Versión 2012.2. Consultado en: www.iucnredlist.org, el 15 de abril 2013.

Centro Regional Universitario Península de Yucatán (CRUPY). 2007. Universidad Autónoma de Chapingo, Mérida, Yucatán, México. Consultado en: http://www.crupy-uach.org. mx el 8 de septiembre 2015.

CHávez, C. 2010. Ecología y conservación del jaguar (Panthera onca) y puma (Puma concolor) en la región de Calakmul y sus implicaciones para la conservación de la Península de Yucatán. Tesis de doctoral. Universidad de Granada. Granada, España. 
De Villa Meza, A. 2006. Áreas prioritarias para la Conservación de los Carnívoros de Oaxaca. Tesis de Maestría, Instituto de Biología, Universidad Nacional Autónoma de México. Ciudad de México, México..

Foerster, C., y C. Vaughan. 2002. Home Range, Habitat Use, and Activity of Baird's tapir in Costa Rica. Programa Regional en Manejo de Vida Silvestre para Mesoamérica y el Caribe. Biotropica 34:423-437.

Folan, W. J., J. Gunn, J. Eaton, y R. Patch. 1983. Paleoclimatological Patterning in Southern Mesoamerica. Journal of Field Archaeology 10:453-468.

Guerra, R. M. 2010. Conocimiento integral del tapir (Tapirus bairdii) con métodos estandarizados de monitoreo biológico en parte de la Selva Maya (México). Informe final. Proyecto realizado para "Memorándum de Entendimiento para la Cooperación en Materia de Conservación de Áreas Protegidas".

Goulart, F. V., N. C. Cáceres, M. E. Graipel, M. A. Tortato, I. R. Ghizoni, JR., y L. G. R. OliveiraSANTOS. 2009. Habitat selection by large mammals in a southern Brazilian Atlantic Forest. Mammalian Biology 74:182-190.

Gunn, J., y R. E. Adams. 1981. Climatic change, culture and civilization in North America. World Archaeology 13:85-100.

Hernández, P. A., C. H. Graham, L. L. Master, y D. L. Albert. 2006. The effect of sample size and species characteristics on performance of different species distribution modeling methods. Ecography 29:773-785.

Hijmans, R. J., S. E. Cameron, J. L. Parra, P. G. Jones, y A. Jarvis. 2005. Very high resolution interpolated climate surfaces for global land areas. International Journal of Climatology 25:1965-1978. Consultado en: http://www.worldclim.org/ el 12 de enero 2013.

Instituto Nacional de Estadística y Goegrafía (INEGI). 1999. Cartas de uso de suelo y vegetación. Consultado en: http://www.inegi.org.mx/geo/contenidos/recnat/usosuelo/ el 25 de marzo 2013

Kappelle, M., y A. D. Brown. 2001. Bosques nublados del Neotrópico. Instituto Nacional de Biodiversidad de Costa Rica. Santo Domingo de Heredia, Costa Rica.

Kauffer, M., y C. L. Villanueva. 2011. Retos de la gestión de una cuenca construida: la península de Yucatán en México. Aqua-lac 3:81-91.

Levin, S. A. 1992. The problem of pattern and scale in ecology. Ecology 73:1943-1976.

Lawton, R. O. 2000. Baird's tapir. Pp. 2342-2343 en Monteverde: ecology and conservation of a tropical cloud forest (Nadkarni, N. M., y N.T. Wheelwright, eds.). Oxford University Press. New York, EE. UU.

Lira-Torres, I., y M. Briones-Salas. 2012. Abundancia relativa y patrones de actividad de los mamíferos de los Chimalapas. Oaxaca, México. Acta Zoológica Mexicana (n. s.) 28:566-585.

Lopez de Casenave, J., L. Marone, F. Jaksic, y P. Camus. 2007. Escalas. Pp. 193-213 en Ecología de Comunidades (Jaksic, F., y L. Marone, eds.). Segunda edición ampliada. Ediciones Universidad Católica de Chile. Santiago de Chile, Chile.

Lugo-Hubp, J., J. Aceves Quesada, y R. Espinosa-Pereña. 1992. Rasgos geomorfológicos mayores de la Península de Yucatán. Instituto de Geología 10:143- 150.

Mendoza Ramírez, E., y J. P. Carbajal Borges. 2011. Avances y perspectivas para la conservación del tapir centroamericano en México. Biodiversitas 99:12-16.

March, I. J. y E. Naranjo. 2005. Orden Perissodactyla. Pp. 495-497. En Los mamíferos silvestres de México (Ceballos, G., y G. Oliva, coords.). CONABIO. Fondo de Cultura Económica. México. 
Mardero, S., E. Nickl, B. Schmook, L. Schneider, J. Rogan, Z. Christman y D. Lawrence. 2011. Sequias en el sur de la Península de Yucatán: análisis de la variabilidad anual y estacional de la precipitación. Investigaciones Geográficas, Boletín del Instituto de Geografía, Universidad Nacional Autónoma de México. México. 78:19-33.

Naranjo, E. 2002. Population ecology and conservation of ungulates in the Lacandon Forest, Mexico. Tesis postdoctoral, Universidad de Florida. Gainesville, EE. UU.

Naranjo, E. 2009. Ecology and Conservation of Baird's tapir in Mexico. Tropical Conservation Science 2:140-158.

Naranjo, E.J., y C.Vaughan. 2000. Ampliación del ámbito altitudinal del tapirCentroamericano (Tapirus bairdii). Revista de Biología Tropical 48:724.

Nolasco, A. L., I. LiRa, y G. Ceballos. 2007. Ampliación en el área de distribución histórica del tapir (Tapirus bairdii) en el Pacífico mexicano. Revista Mexicana de Mastozoología 11:91-94.

Nolasco, A. 2009. Distribución actual y estado de conservación del tapir centroamericano Tapirus bairdii Gill, 1985 (Perissodactyla: Tapiridae) en México. Tesis de licenciatura, Facultad de ciencias, Universidad Nacional Autónoma de México. Ciudad de México, México.

Pedlar, J. H., L. Fahrig, y H. G. Merriam. 1997. Raccoon habitat use at two spatial scales. The Journal of Wildlife Management 61:102-112.

Pérez-Solano, L. A. 2011. Caracterización del hábitat del venado temazate rojo a dos escalas espaciales en la región montañosa de Oaxaca, México. Tesis de Maestría, Instituto de Ecología. Xalapa, México.

Phillips, S., R. Anderson, y R. Schapire. 2006. Maximum entropy modeling of species geographic distributions. Ecological modelling 190:231-259

Reid, F. A. 1997. A field guide to the mammals of Central America and Southeast Mexico. Oxford University Press. New York, EE.UU.

Robinson, J., Y K. REDFoRD. 1994. Measuring the sustainability of hunting in tropical forests. Oryx 28:249-256.

$\mathrm{SAAB}_{\mathbf{A}}$ V. 1999. Importance of spatial scale to habitat use by breeding birds in riparian forest: a hierarchical analysis. Ecological applications 9:135-151.

Secretaria de Medio Ambiente y Recursos Naturales (SEMARNAT). 2010. Norma Oficial Mexicana NOM-059-SEMARNAT-2010. Protección ambiental-especies nativas de México de flora y fauna silvestres- categorías de riesgo y especificaciones para su inclusión, exclusión o cambio- lista de especies en riesgo. Diario Oficial de la Federación, 30 de diciembre de 2010:1-56.

Tobler, M. W., E. J. Naranjo, y I. LiRa-Torres. 2006. Habitat preference, feeding habits and conservation of Baird's tapir in Neotropical montane oak forests. Pp. 347-361 en Ecology and conservation of Neotropical montane oak forests (Kaapelle, M. eds). Springer-Verlag. Munich, Germany.

Turner, M., R. H. Gardner. y R. V. O’Neill. 2001. Landscape ecology in theory and practice: pattern and process. Springer-Verlag. New York, EE. UU.

Unión Internacional para la Conservación de la Naturaleza, lista Roja de Especies Amenazadas. 2013. Consultado en: http://www.iucnredlist.org el 01/04/2013

Vaca, R. A., J. G. Duncan, L. Cayuela, J. Hewson, y M. Steininger. 2012. Evidence of Incipient Forest Transition in Southern Mexico. PLoS ONE 7: e42309.

Vester, H., D. Lawrence, J. Eastmant, R. Turner, S. Calmé, R. Dickson, C. Pozo, y F. Sangermano. 2007. Land change in the southern Yucatan and Calakmul Biosphere Reserve: effects on habitat and biodiversity. The Ecological Society of America 174:989-1003. 
Young, N., L. Carter, y P. Evangelista. 2011. A MaxEnt Model v3.3.3e Tutorial (ArcGIS v10). Creado en: The Natural Resource Ecology Laboratory at Colorado State University and the National Institute of Invasive Species Science. Colorado State University. Denver, EE. UU.

Summited: April 27, 2015

Review: May 12, 2015

Accepted: September 9, 2015

Associated editor: Sergio Solari 


\section{Apéndice I}

Fuentes de registros de tapir centroamericano en la península de Yucatán para generar el mapa de distribución potencial.

Colección Mastozoológica de El Colegio de la Frontera Sur, unidad Chetumal, Quintana Roo, México. Datos proporcionados por el curador, Biólogo Enrique Escobedo Cabrera.

Datos proporcionados por la dirección de la Reserva de la Biosfera de Sian Ka \an. Cancún, Benito Juárez, Quintana Roo, México, mediante el oficio núm. F00.9.DRBSK-080/13.

Datos proporcionados por Jorge Gómez Poot. 2013. Subdirección Parque Natural Arrecifes de Xcalak, Quintana Roo, México.

- Base de datos recopilada para la investigación: Zúñiga-Fuentes, H. 2008. Distribución y conocimiento ecológico local del tapir centroamericano (Tapirus bairdii) en el estado de Campeche. Tesis de licenciatura. El Colegio de la Frontera Sur, Campeche, México.

Base de datos recopilada para la investigación: Nolasco, L. 2009. Distribución actual y estado de conservación del tapir centroamericano Tapirus bairdii, Gill, 1865 (perissodactyla: tapiridae) en México. Tesis de licenciatura. Universidad Nacional Autónoma de México. Ciudad de México, México

Base de datos recopilada para la investigación: Mendoza, E., T. L. Fuller, H. A. Thomassen, W. Buerman, D. Ramírez-Mejía y T. B. Smith. 2013. A preliminary assessment of the effectiveness of the Mesoamerican Biological Corridor for protecting potential Baird's tapir (Tapirus bairdii) habitat in southern Mexico. Integrative Zoology 8:34-46.

Datos obtenidos en la investigación: Pérez-Cortez, S.. y E. S. Matus-Pérez. 2010. El tapir Tapirus bairdii en la región sureste del Área de Protección de Flora y Fauna Bala'an Ka'ax, Quintana Roo. Therya 1:137-144.

Datos obtenidos en la investigación: Serrano, I. 2013. Foto trampeo de mamíferos y aves asociados a las aguadas de la región sur de Calakmul, Campeche, México. Tesis de licenciatura. Universidad Autónoma de Campeche, Campeche, México.

Datos obtenidos en la investigación: Ramírez-Barajas P. J., N. Torrescano-Valle, M. Sanvicente López, A.Tuz Novelo, y M. Tuz Novelo. 2010. Evaluación del impacto del huracán Dean sobre la fauna y su hábitat en el complejo Sian Ka'an y ejidos de influencia, Quintana Roo, México. Servicios y Beneficios Ambientales SEYBA SC de RL- COMPACT. Global Environment Facility. Programa de pequeñas donaciones PPD-PNUD. Informe Final.

Datos obtenidos en la investigación: Pérez-Cortes S., P. Enríquez, D. Sima-Panti, R. ReynaHurtado, y E. Naranjo. 2012. Influencia de la disponibilidad de agua en la presencia y abundancia de Tapirus bairdii en la selva de Calakmul, Campeche, México. Revista Mexicana de Biodiversidad 83: 753-761.

Datos obtenidos en la investigación: Naranjo, E. J., S. A. Amador, F. Falconi, y R. Reyna. 2013. Conocimiento integral del tapir (Tapirus bairdii) y el pecarí de labios blancos (Tayassu pecari) en México. Informe técnico final del proyecto HK014. Comisión Nacional para el Conocimiento y Uso de la Biodiversidad. Ciudad de México, México.

Datos obtenidos en la investigación: Naranjo, E. J., S. A. Amador, F. Falconi, y R. Reyna. 2015. Distribución, abundancia y amenazas a las poblaciones de tapir (Tapirus bairdii) y pecarí de labios blancos (Tayassu pecari) en México. Therya 6:227-249.

Datos obtenidos en la investigación: O'Farrill G. 2010. The ecological causes and consequences of the movement of Baird's tapir (Tapirus bairdii). Tesis de doctorado. McGill University, Montreal, Quebec, Canada. 
Datos obtenidos en la investigación: Carrillo-Reyna, N., R. Reyna-Hurtado, y B. Schmook. 2015. Abundancia relativa y selección de hábitat de Tapirus bairdii en las reservas de Calakmul y Balam Kú, Campeche, México. Revista Mexicana de Biodiversidad 86:202207.

Datos proporcionados por M .C. Mauro San Vicente. El Colegio de la Frontera Sur, Chetumal, Quintana Roo, México.

Datos proporcionados por el veterinario zootecnista Jonathan Pérez Flores. El Colegio de la Frontera Sur, Chetumal, Quintana Roo, México. 


\section{Apéndice II}

\begin{tabular}{|c|c|c|c|}
\hline Localidad & Longitud & Latitud & Referencia \\
\hline Nadzcan, Campeche & $-89.894580^{\circ}$ & $18.549320^{\circ}$ & Zuñiga 2008 \\
\hline Calakmul, Campeche & $-89.745480^{\circ}$ & $18.158550^{\circ}$ & Zuñiga 2008 \\
\hline Calakmul, Campeche & $-89.779830^{\circ}$ & $18.314980^{\circ}$ & Zuñiga 2008 \\
\hline Calakmul, Campeche & $-89.820070^{\circ}$ & $18.124870^{\circ}$ & Zuñiga 2008 \\
\hline Balam Kú, Campeche & $-89.893840^{\circ}$ & $18.549420^{\circ}$ & Zuñiga 2008 \\
\hline Balam Kú, Campeche & $-89.891020^{\circ}$ & $18.550170^{\circ}$ & Zuñiga 2008 \\
\hline Balam Kú, Campeche & $-89.894450^{\circ}$ & $18.549310^{\circ}$ & Zuñiga 2008 \\
\hline Balam Kú, Campeche & $-89.858730^{\circ}$ & $18.309840^{\circ}$ & Zuñiga 2008 \\
\hline Nadzcan-Balam Kú & $-89.894540^{\circ}$ & $18.549480^{\circ}$ & Zuñiga 2008 \\
\hline Hopelchen, Campeche & $-89.327990^{\circ}$ & $19.081610^{\circ}$ & Zuñiga 2008 \\
\hline Xbonil, Campeche & $-89.550300^{\circ}$ & $18.321800^{\circ}$ & Zuñiga 2008 \\
\hline 20 de noviembre, Campeche & $-89.202400^{\circ}$ & $18.252600^{\circ}$ & Zuñiga 2008 \\
\hline 20 de noviembre, Campeche & $-89.161930^{\circ}$ & $18.404800^{\circ}$ & Zuñiga 2008 \\
\hline 22 de noviembre, Campeche & $-89.093400^{\circ}$ & $18.491700^{\circ}$ & Zuñiga 2008 \\
\hline Balam Kin, Campeche & $-90.035889^{\circ}$ & $18.902833^{\circ}$ & Zuñiga 2008 \\
\hline Balam Kin, Campeche & $-90.047500^{\circ}$ & $18.895944^{\circ}$ & Zuñiga 2008 \\
\hline Balam Kú, Campeche & $-89.846411^{\circ}$ & $18.607503^{\circ}$ & Zuñiga 2008 \\
\hline Calakmul, Campeche & $-89.885833^{\circ}$ & $18.346833^{\circ}$ & Zuñiga 2008 \\
\hline Calakmul, Campeche & $-89.859389^{\circ}$ & $18.809678^{\circ}$ & Zuñiga 2008 \\
\hline Zoh Laguna, Campeche & $-89.417806^{\circ}$ & $18.583472^{\circ}$ & Zuñiga 2008 \\
\hline Calakmul, Campeche & $-89.552250^{\circ}$ & $17.951111^{\circ}$ & Zuñiga 2008 \\
\hline Calakmul, Campeche & $-89.451583^{\circ}$ & $18.099611^{\circ}$ & Zuñiga 2008 \\
\hline Calakmul, Campeche & $-89.498917^{\circ}$ & $18.193389^{\circ}$ & Zuñiga 2008 \\
\hline 20 de noviembre, Campeche & $-89.252778^{\circ}$ & $18.396472^{\circ}$ & Zuñiga 2008 \\
\hline 20 de noviembre, Campeche & $-89.305942^{\circ}$ & $18.343917^{\circ}$ & Zuñiga 2008 \\
\hline Nuevo Becal, Campeche & $-89.159000^{\circ}$ & $18.709861^{\circ}$ & Zuñiga 2008 \\
\hline Hopelchen, Campeche & $-89.436528^{\circ}$ & $18.803472^{\circ}$ & Zuñiga 2008 \\
\hline Hopelchen, Campeche & $-89.424806^{\circ}$ & $18.799833^{\circ}$ & Zuñiga 2008 \\
\hline Xmaben, Campeche & $-89.173306^{\circ}$ & $19.185083^{\circ}$ & Zuñiga 2008 \\
\hline Calakmul, Campeche & $-89.856830^{\circ}$ & $18.316100^{\circ}$ & Zuñiga 2008 \\
\hline Calakmul, Campeche & $-89.745480^{\circ}$ & $18.158550^{\circ}$ & Zuñiga 2008 \\
\hline Calakmul, Campeche & $-89.779830^{\circ}$ & $18.314980^{\circ}$ & Zuñiga 2008 \\
\hline Calakmul, Campeche & $-89.816780^{\circ}$ & $18.125740^{\circ}$ & Zuñiga 2008 \\
\hline Balam Kú, Campeche & $-89.893840^{\circ}$ & $18.549420^{\circ}$ & Zuñiga 2008 \\
\hline Calakmul, Campeche & $-89.485710^{\circ}$ & $18.314870^{\circ}$ & Zuñiga 2008 \\
\hline Calakmul, Campeche & $-89.566270^{\circ}$ & $18.327140^{\circ}$ & Zuñiga 2008 \\
\hline
\end{tabular}




\begin{tabular}{|c|c|c|c|}
\hline Localidad & Longitud & Latitud & Referencia \\
\hline Calakmul, Campeche & $-89.566730^{\circ}$ & $18.328270^{\circ}$ & Zuñiga 2008 \\
\hline Calakmul, Campeche & $-89.566560^{\circ}$ & $18.327920^{\circ}$ & Zuñiga 2008 \\
\hline Balam Kin, Campeche & $-90.014889^{\circ}$ & $19.031503^{\circ}$ & Zuñiga 2008 \\
\hline Balam Kin, Campeche & $-90.014744^{\circ}$ & $19.031511^{\circ}$ & Zuñiga 2008 \\
\hline Balam Kin, Campeche & $-90.014994^{\circ}$ & $19.031894^{\circ}$ & Zuñiga 2008 \\
\hline Balam Kin, Campeche & $-90.015333^{\circ}$ & $19.032644^{\circ}$ & Zuñiga 2008 \\
\hline Chilambalam, Campeche & $-90.252139^{\circ}$ & $18.948611^{\circ}$ & Zuñiga 2008 \\
\hline Calakmul, Campeche & $-89.816639^{\circ}$ & $18.123496^{\circ}$ & Zuñiga 2008 \\
\hline Calakmul, Campeche & $-89.741044^{\circ}$ & $18.180601^{\circ}$ & Zuñiga 2008 \\
\hline Calakmul, Campeche & $-89.330193^{\circ}$ & $19.139376^{\circ}$ & Zuñiga 2008 \\
\hline Calakmul, Campeche & $-89.899641^{\circ}$ & $18.544296^{\circ}$ & Zuñiga 2008 \\
\hline Calakmul, Campeche & $-89.858246^{\circ}$ & $18.602813^{\circ}$ & Zuñiga 2008 \\
\hline 20 de noviembre, Campeche & $-89.312594^{\circ}$ & $18.471294^{\circ}$ & Zuñiga 2008 \\
\hline 20 de noviembre, Campeche & $-89.359778^{\circ}$ & $18.420024^{\circ}$ & Zuñiga 2008 \\
\hline 20 de noviembre, Campeche & $-89.353279^{\circ}$ & $18.313372^{\circ}$ & Zuñiga 2008 \\
\hline 20 de noviembre, Campeche & $-89.312982^{\circ}$ & $18.380740^{\circ}$ & Zuñiga 2008 \\
\hline Nuevo Becal, Campeche & $-89.266261^{\circ}$ & $18.594180^{\circ}$ & Zuñiga 2008 \\
\hline Calakmul, Campeche & $-89.885850^{\circ}$ & $18.365580^{\circ}$ & Zuñiga 2008 \\
\hline Nuevo Becal, Campeche & $-89.180444^{\circ}$ & $18.741407^{\circ}$ & Zuñiga 2008 \\
\hline Calakmul, Campeche & $-89.686626^{\circ}$ & $17.914600^{\circ}$ & Zuñiga 2008 \\
\hline Calakmul, Campeche & $-89.494940^{\circ}$ & $18.076933^{\circ}$ & Zuñiga 2008 \\
\hline Calakmul, Campeche & $-89.487338^{\circ}$ & $18.206418^{\circ}$ & Zuñiga 2008 \\
\hline 20 de noviembre, Campeche & $-89.243678^{\circ}$ & $18.351880^{\circ}$ & Zuñiga 2008 \\
\hline Calakmul, Campeche & $-89.802654^{\circ}$ & $18.267127^{\circ}$ & Zuñiga 2008 \\
\hline Zoh Laguna, Campeche & $-89.369577^{\circ}$ & $18.611478^{\circ}$ & Zuñiga 2008 \\
\hline Calakmul, Campeche & $-89.395404^{\circ}$ & $18.798928^{\circ}$ & Zuñiga 2008 \\
\hline Nuevo Becal, Campeche & $-89.236127^{\circ}$ & $18.691766^{\circ}$ & Zuñiga 2008 \\
\hline Nuevo Becal, Campeche & $-89.240000^{\circ}$ & $18.651119^{\circ}$ & Zuñiga 2008 \\
\hline Calakmul, Campeche & $-89.827757^{\circ}$ & $18.328294^{\circ}$ & Zuñiga 2008 \\
\hline Calakmul, Campeche & $-89.931145^{\circ}$ & $18.342668^{\circ}$ & Zuñiga 2008 \\
\hline Calakmul, Campeche & $-89.836851^{\circ}$ & $18.327012^{\circ}$ & Zuñiga 2008 \\
\hline Calakmul, Campeche & $-89.876858^{\circ}$ & $18.312498^{\circ}$ & Zuñiga 2008 \\
\hline Calakmul, Campeche & $-90.009488^{\circ}$ & $18.118525^{\circ}$ & Zuñiga 2008 \\
\hline Calakmul, Campeche & $-90.132377^{\circ}$ & $18.143718^{\circ}$ & Zuñiga 2008 \\
\hline Calakmul, Campeche & $-90.071263^{\circ}$ & $17.883583^{\circ}$ & Zuñiga 2008 \\
\hline Calakmul, Campeche & $-90.527898^{\circ}$ & $18.765723^{\circ}$ & Zuñiga 2008 \\
\hline
\end{tabular}




\begin{tabular}{|c|c|c|c|}
\hline Localidad & Longitud & Latitud & Referencia \\
\hline Calakmul, Campeche & $-90.005009^{\circ}$ & $19.206381^{\circ}$ & Zuñiga 2008 \\
\hline Chilambalam, Campeche & $-90.271157^{\circ}$ & $18.903546^{\circ}$ & Zuñiga 2008 \\
\hline Calakmul, Campeche & $-89.795331^{\circ}$ & $18.141152^{\circ}$ & Zuñiga 2008 \\
\hline Nuevo Becal, Campeche & $-89.202166^{\circ}$ & $18.582159^{\circ}$ & Zuñiga 2008 \\
\hline Calakmul, Campeche & $-89.828998^{\circ}$ & $18.523833^{\circ}$ & Zuñiga 2008 \\
\hline Calakmul, Campeche & $-90.053523^{\circ}$ & $18.583955^{\circ}$ & Zuñiga 2008 \\
\hline Calakmul, Campeche & $-90.331405^{\circ}$ & $18.022963^{\circ}$ & Zuñiga 2008 \\
\hline Calakmul, Campeche & $-89.815957^{\circ}$ & $18.125149^{\circ}$ & Serrano 2013 \\
\hline Calakmul, Campeche & $-89.783177^{\circ}$ & $18.105995^{\circ}$ & Serrano 2013 \\
\hline Calakmul, Campeche & $-89.746308^{\circ}$ & $18.185555^{\circ}$ & Serrano 2013 \\
\hline Calakmul, Campeche & $-89.752617^{\circ}$ & $18.205277^{\circ}$ & Serrano 2013 \\
\hline Calakmul, Campeche & $-89.857199^{\circ}$ & $18.308189^{\circ}$ & Serrano 2013 \\
\hline Calakmul, Campeche & $-89.857091^{\circ}$ & $18.307919^{\circ}$ & Serrano 2013 \\
\hline Calakmul, Campeche & $-89.832361^{\circ}$ & $18.310239^{\circ}$ & Serrano 2013 \\
\hline Calakmul, Campeche & $-89.885557^{\circ}$ & $18.366722^{\circ}$ & Serrano 2013 \\
\hline San Antonio Cumpich, Campeche & $-89.927250^{\circ}$ & $20.246080^{\circ}$ & Nolasco 2009 \\
\hline Xpujil, Campeche & $-89.433330^{\circ}$ & $18.733610^{\circ}$ & Nolasco 2009 \\
\hline Constitucion, Campeche & $-90.131670^{\circ}$ & $18.807220^{\circ}$ & Nolasco 2009 \\
\hline Constitución, Campeche & $-90.146110^{\circ}$ & $17.945830^{\circ}$ & Nolasco 2009 \\
\hline Calakmul, Campeche & $-89.796270^{\circ}$ & $17.817760^{\circ}$ & Nolasco 2009 \\
\hline Balam Kin, Campeche & $-90.014950^{\circ}$ & $19.031560^{\circ}$ & Nolasco 2009 \\
\hline Calakmul, Campeche & $-89.815556^{\circ}$ & $18.122500^{\circ}$ & Nolasco 2009 \\
\hline Xbonil, Campeche & $-90.149722^{\circ}$ & $18.816667^{\circ}$ & Nolasco 2009 \\
\hline Felipe Carrillo Puerto, Quintana Roo & $-87.676110^{\circ}$ & $19.798610^{\circ}$ & Nolasco 2009 \\
\hline X-Hazil, Quintana Roo & $-87.959720^{\circ}$ & $19.282220^{\circ}$ & Nolasco 2009 \\
\hline Chumpon, Quintana Roo & $-87.580560^{\circ}$ & $19.817780^{\circ}$ & Nolasco 2009 \\
\hline Andres Quintana Roo, Quintana Roo & $-87.888890^{\circ}$ & $19.159720^{\circ}$ & Nolasco 2009 \\
\hline Chumpón, Quintana Roo & $-87.783330^{\circ}$ & $19.616670^{\circ}$ & Nolasco 2009 \\
\hline Reserva de la Biosfera de Sian Kaan, Quintana Roo & $-87.783610^{\circ}$ & $19.743890^{\circ}$ & Nolasco 2009 \\
\hline Uaimyl, Quintana Roo & $-87.780000^{\circ}$ & $19.070000^{\circ}$ & Nolasco 2009 \\
\hline Xcalak, Quintana Roo & $-87.833330^{\circ}$ & $18.266670^{\circ}$ & Nolasco 2009 \\
\hline Chiquilá, Quintana Roo & $-87.253890^{\circ}$ & $21.420556^{\circ}$ & Nolasco 2009 \\
\hline Chiquila, Quintana Roo & $-87.520000^{\circ}$ & $21.470000^{\circ}$ & Nolasco 2009 \\
\hline Nuevo Becal, Campeche & $-89.301670^{\circ}$ & $18.622222^{\circ}$ & Nolasco 2009 \\
\hline 20 de noviembre, Campeche & $-89.281940^{\circ}$ & $18.355833^{\circ}$ & Nolasco 2009 \\
\hline Majahual, Quintana Roo & $-87.778890^{\circ}$ & $18.496667^{\circ}$ & Nolasco 2009 \\
\hline
\end{tabular}




\begin{tabular}{|c|c|c|c|}
\hline Localidad & Longitud & Latitud & Referencia \\
\hline Vigia chico, Quintana Roo & $-87.755830^{\circ}$ & $19.759722^{\circ}$ & Nolasco 2009 \\
\hline Caobas, Quintana Roo & $-88.984812^{\circ}$ & $18.457044^{\circ}$ & Nolasco 2009 \\
\hline Chiquilá, Quintana roo & $-87.412500^{\circ}$ & $21.385278^{\circ}$ & Nolasco 2009 \\
\hline Costa maya, Quintana Roo & $-87.849444^{\circ}$ & $18.887222^{\circ}$ & Nolasco 2009 \\
\hline Quintana Roo & $-90.103000^{\circ}$ & $17.884900^{\circ}$ & Mendoza 2013 \\
\hline Quintana Roo & $-89.217300^{\circ}$ & $19.236800^{\circ}$ & Mendoza 2013 \\
\hline Quintana Roo & $-89.783100^{\circ}$ & $18.104300^{\circ}$ & Mendoza 2013 \\
\hline Quintana Roo & $-90.096200^{\circ}$ & $18.010600^{\circ}$ & Mendoza 2013 \\
\hline Quintana Roo & $-87.787400^{\circ}$ & $19.720500^{\circ}$ & Mendoza 2013 \\
\hline Quintana Roo & $-87.822900^{\circ}$ & $19.713900^{\circ}$ & Mendoza 2013 \\
\hline Quintana Roo & $-87.744700^{\circ}$ & $19.907200^{\circ}$ & Mendoza 2013 \\
\hline Quintana Roo & $-87.740500^{\circ}$ & $19.911500^{\circ}$ & Mendoza 2013 \\
\hline Quintana Roo & $-87.735700^{\circ}$ & $19.916400^{\circ}$ & Mendoza 2013 \\
\hline Quintana Roo & $-87.724300^{\circ}$ & $19.927600^{\circ}$ & Mendoza 2013 \\
\hline Quintana Roo & $-87.622100^{\circ}$ & $19.786600^{\circ}$ & Mendoza 2013 \\
\hline Quintana Roo & $-87.750700^{\circ}$ & $19.901000^{\circ}$ & Mendoza 2013 \\
\hline Quintana Roo & $-87.765100^{\circ}$ & $19.886600^{\circ}$ & Mendoza 2013 \\
\hline Quintana Roo & $-87.770200^{\circ}$ & $19.881600^{\circ}$ & Mendoza 2013 \\
\hline Quintana Roo & $-87.770500^{\circ}$ & $19.880600^{\circ}$ & Mendoza 2013 \\
\hline Quintana Roo & $-87.770900^{\circ}$ & $19.880700^{\circ}$ & Mendoza 2013 \\
\hline Campeche & $-89.193900^{\circ}$ & $18.681700^{\circ}$ & O'Farrill $2010^{*}$ \\
\hline Campeche & $-89.815500^{\circ}$ & $18.129900^{\circ}$ & O'Farrill $2010^{*}$ \\
\hline Campeche & $-89.280700^{\circ}$ & $18.636100^{\circ}$ & O'Farrill $2010^{*}$ \\
\hline Campeche & $-89.177600^{\circ}$ & $18.738700^{\circ}$ & O’Farrill 2010* \\
\hline Campeche & $-89.234500^{\circ}$ & $18.628900^{\circ}$ & O’Farrill 2010* \\
\hline Campeche & $-89.478400^{\circ}$ & $18.247500^{\circ}$ & O’Farrill 2010* \\
\hline Campeche & $89.513400^{\circ}$ & $18.240100^{\circ}$ & O'Farrill 2010* \\
\hline Campeche & $-89.514500^{\circ}$ & $18.206900^{\circ}$ & O'Farrill 2010* \\
\hline Campeche & $-89.209900^{\circ}$ & $18.674900^{\circ}$ & O'Farrill $2010^{*}$ \\
\hline Campeche & $-89.280100^{\circ}$ & $18.638000^{\circ}$ & O'Farrill 2010* \\
\hline Campeche & $-89.256300^{\circ}$ & $18.692600^{\circ}$ & O’Farrill 2010* \\
\hline Campeche & $-89.252500^{\circ}$ & $18.691500^{\circ}$ & O’Farrill 2010* \\
\hline Campeche & $-89.233700^{\circ}$ & $18.736700^{\circ}$ & O'Farrill 2010* \\
\hline Campeche & $-89.249700^{\circ}$ & $18.689600^{\circ}$ & O'Farrill $2010^{*}$ \\
\hline Campeche & $-89.254900^{\circ}$ & $18.646600^{\circ}$ & O’Farrill 2010* \\
\hline Campeche & $-89.752600^{\circ}$ & $18.205300^{\circ}$ & Perez-Cortez 2011* \\
\hline
\end{tabular}




\begin{tabular}{|c|c|c|c|}
\hline Localidad & Longitud & Latitud & Referencia \\
\hline Campeche & $-89.719600^{\circ}$ & $18.074000^{\circ}$ & Perez-Cortez 2011* \\
\hline Campeche & $-90.107600^{\circ}$ & $17.843300^{\circ}$ & Perez-Cortez 2011* \\
\hline Campeche & $-90.096700^{\circ}$ & $18.013400^{\circ}$ & Perez-Cortez 2011* \\
\hline Campeche & $-89.857100^{\circ}$ & $18.307900^{\circ}$ & Perez-Cortez 2011* \\
\hline Campeche & $-89.778100^{\circ}$ & $18.308300^{\circ}$ & Perez-Cortez 2011* \\
\hline Campeche & $-90.002100^{\circ}$ & $17.903100^{\circ}$ & Perez-Cortez 2011* \\
\hline Campeche & $-90.090800^{\circ}$ & $17.855400^{\circ}$ & Perez-Cortez 2011* \\
\hline Calakmul, Campeche & $-88.464416^{\circ}$ & $19.358202^{\circ}$ & San Vicente 2005-2012 \\
\hline Calakmul, Campeche & $-89.804522^{\circ}$ & $18.088189^{\circ}$ & San Vicente 2005-2012 \\
\hline Nuevo Becal, Campeche & $-89.210407^{\circ}$ & $18.670156^{\circ}$ & San Vicente 2005-2012 \\
\hline Nuevo Becal, Campeche & $-89.208271^{\circ}$ & $18.674218^{\circ}$ & San Vicente 2005-2012 \\
\hline Calakmul, Campeche & $-89.804147^{\circ}$ & $18.075913^{\circ}$ & San Vicente 2005-2012 \\
\hline Conhuas, Campeche & $-89.917052^{\circ}$ & $18.530716^{\circ}$ & San Vicente 2005-2012 \\
\hline Bacalar, Quintana Roo & $-88.114515^{\circ}$ & $19.165249^{\circ}$ & San Vicente 2005-2012 \\
\hline Bacalar, Quintana Roo & $-88.422131^{\circ}$ & $19.213101^{\circ}$ & San Vicente 2005-2012 \\
\hline Bacalar, Quintana Roo & $-88.316265^{\circ}$ & $18.767890^{\circ}$ & San Vicente 2005-2012 \\
\hline Caobas, Quintana Roo & $-89.137315^{\circ}$ & $18.460737^{\circ}$ & San Vicente 2005-2012 \\
\hline Bacalar, Quintana Roo & $-88.175624^{\circ}$ & $18.822727^{\circ}$ & San Vicente 2005-2012 \\
\hline Bacalar, Quintana Roo & $-87.852627^{\circ}$ & $18.307949^{\circ}$ & Perez-Flores 2012 \\
\hline Bacalar, Quintana Roo & $-87.788927^{\circ}$ & $18.459485^{\circ}$ & Perez-Flores 2012 \\
\hline Balan Ka'ax, Quintana Roo & $-88.936277^{\circ}$ & $19.236352^{\circ}$ & Perez-Cortez y Matus-Perez 2010 \\
\hline Balan Ka'ax, Quintana Roo & $-88.932078^{\circ}$ & $19.240028^{\circ}$ & Perez-Cortez y Matus-Perez 2010 \\
\hline Balan Ka'ax, Quintana Roo & $-88.932387^{\circ}$ & $19.241244^{\circ}$ & Perez-Cortez y Matus-Perez 2010 \\
\hline Balan Ka'ax, Quintana Roo & $-88.917598^{\circ}$ & $19.250261^{\circ}$ & Perez-Cortez y Matus-Perez 2010 \\
\hline Balan Ka'ax, Quintana Roo & $-88.892038^{\circ}$ & $19.259913^{\circ}$ & Perez-Cortez y Matus-Perez 2010 \\
\hline Balan Ka'ax, Quintana Roo & $-88.888270^{\circ}$ & $19.259853^{\circ}$ & Perez-Cortez y Matus-Perez 2010 \\
\hline Balam K'aax & $-88.890618^{\circ}$ & $19.268040^{\circ}$ & Perez-Cortez y Matus-Perez 2010 \\
\hline Balam K'aax & $-88.890746^{\circ}$ & $19.265916^{\circ}$ & Perez-Cortez y Matus-Perez 2010 \\
\hline Balam K'aax & $-88.889531^{\circ}$ & $19.262803^{\circ}$ & Perez-Cortez y Matus-Perez 2010 \\
\hline Balam K'aax & $-88.892113^{\circ}$ & $19.259840^{\circ}$ & Perez-Cortez y Matus-Perez 2010 \\
\hline Balam K'aax & $-88.898166^{\circ}$ & $19.264240^{\circ}$ & Perez-Cortez y Matus-Perez 2010 \\
\hline Balam K'aax & $-88.902571^{\circ}$ & $19.279172^{\circ}$ & Perez-Cortez y Matus-Perez 2010 \\
\hline Balam K'aax & $-88.914051^{\circ}$ & $19.283072^{\circ}$ & Perez-Cortez y Matus-Perez 2010 \\
\hline Balam K'aax & $-88.923418^{\circ}$ & $19.286018^{\circ}$ & Perez-Cortez y Matus-Perez 2010 \\
\hline Balam K'aax & $-88.890587^{\circ}$ & $19.268637^{\circ}$ & Perez-Cortez y Matus-Perez 2010 \\
\hline Balam K'aax & $-88.124612^{\circ}$ & $19.306356^{\circ}$ & Perez-Cortez y Matus-Perez 2010 \\
\hline
\end{tabular}




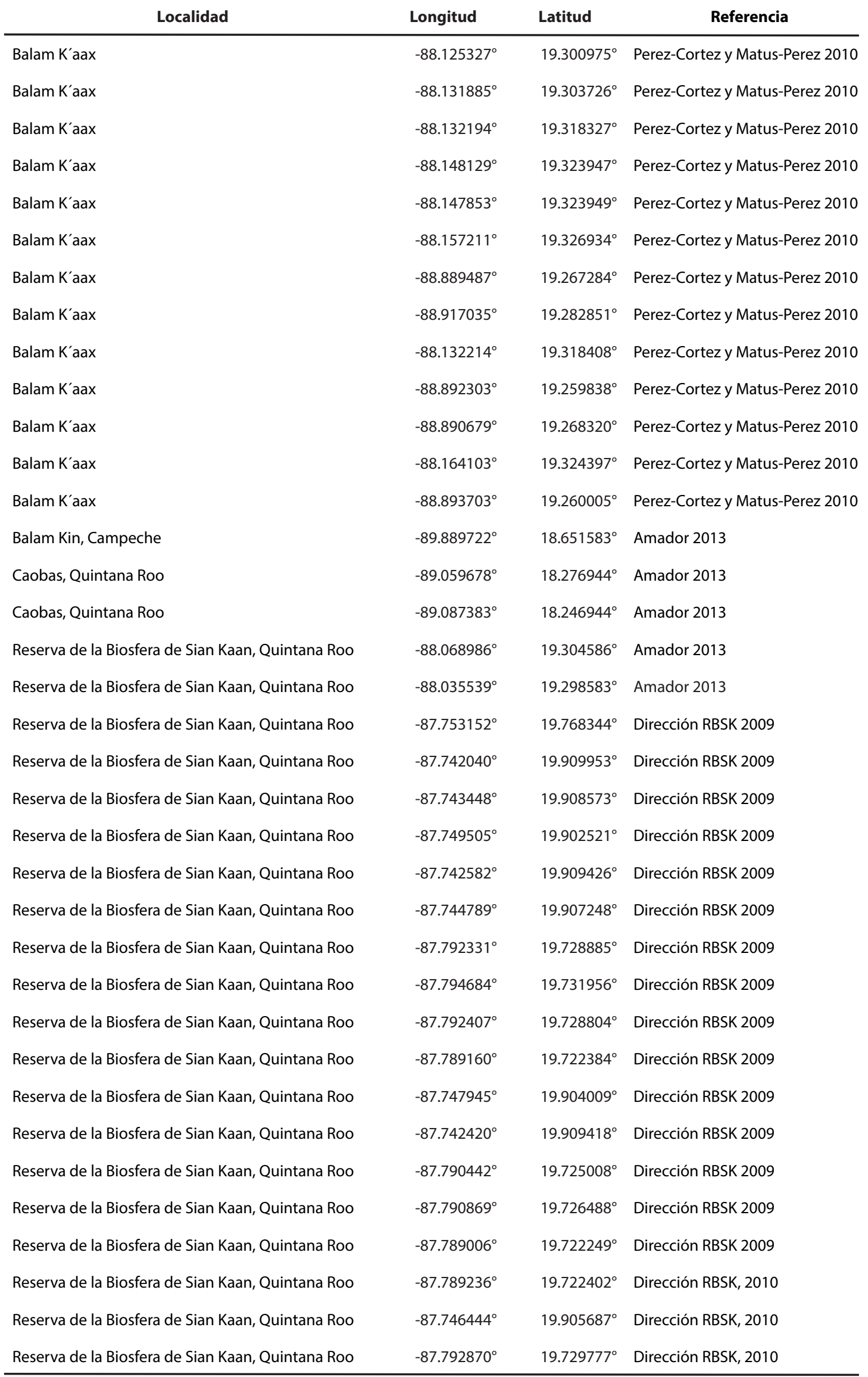




\begin{tabular}{|c|c|c|c|}
\hline Localidad & Longitud & Latitud & Referencia \\
\hline Reserva de la Biosfera de Sian Kaan, Quintana Roo & $-87.851127^{\circ}$ & $19.691567^{\circ}$ & Dirección RBSK, 2012 \\
\hline Xcalak, Quintana Roo & $-87.758509^{\circ}$ & $18.568116^{\circ}$ & Gomez Poot 2005 \\
\hline Xcalak, Quintana Roo & $-87.747832^{\circ}$ & $18.592936^{\circ}$ & Gomez Poot 2005 \\
\hline Xcalak, Quintana Roo & $-87.887148^{\circ}$ & $18.848369^{\circ}$ & Gomez Poot 2005 \\
\hline Xcalak, Quintana Roo & $-87.871370^{\circ}$ & $18.749233^{\circ}$ & Gomez Poot 2005 \\
\hline Xcalak, Quintana Roo & $-87.835089^{\circ}$ & $18.319297^{\circ}$ & Gomez Poot 2005 \\
\hline Calakmul-Balam Kú, Campeche & $-89.865460^{\circ}$ & $18.328164^{\circ}$ & Carrillo 2012 \\
\hline Calakmul-Balam Kú, Campeche & $-89.843863^{\circ}$ & $18.326844^{\circ}$ & Carrillo 2012 \\
\hline Calakmul-Balam Kú, Campeche & $-89.866658^{\circ}$ & $18.307920^{\circ}$ & Carrillo 2012 \\
\hline Calakmul-Balam Kú, Campeche & $-89.871389^{\circ}$ & $18.318314^{\circ}$ & Carrillo 2012 \\
\hline Calakmul-Balam Kú, Campeche & $-89.872284^{\circ}$ & $18.619411^{\circ}$ & Carrillo 2012 \\
\hline Calakmul-Balam Kú, Campeche & $-89.870189^{\circ}$ & $18.331410^{\circ}$ & Carrillo 2012 \\
\hline Calakmul-Balam Kú, Campeche & $-89.792415^{\circ}$ & $18.133528^{\circ}$ & Carrillo 2012 \\
\hline Calakmul-Balam Kú, Campeche & $-89.792435^{\circ}$ & $18.134205^{\circ}$ & Carrillo 2012 \\
\hline Calakmul-Balam Kú, Campeche & $-89.792987^{\circ}$ & $18.135082^{\circ}$ & Carrillo 2012 \\
\hline Calakmul-Balam Kú, Campeche & $-89.848785^{\circ}$ & $18.327213^{\circ}$ & Carrillo 2012 \\
\hline Calakmul-Balam Kú, Campeche & $-89.879900^{\circ}$ & $18.610545^{\circ}$ & Carrillo 2012 \\
\hline Calakmul-Balam Kú, Campeche & $-89.865100^{\circ}$ & $18.598718^{\circ}$ & Carrillo 2012 \\
\hline Calakmul-Balam Kú, Campeche & $-89.872774^{\circ}$ & $18.618130^{\circ}$ & Carrillo 2012 \\
\hline Calakmul-Balam Kú, Campeche & $-89.876460^{\circ}$ & $18.614887^{\circ}$ & Carrillo 2012 \\
\hline Calakmul-Balam Kú, Campeche & $-89.869412^{\circ}$ & $18.552008^{\circ}$ & Carrillo 2012 \\
\hline Calakmul-Balam Kú, Campeche & $-89.938666^{\circ}$ & $18.556287^{\circ}$ & Carrillo 2012 \\
\hline Calakmul-Balam Kú, Campeche & $-89.889455^{\circ}$ & $18.553255^{\circ}$ & Carrillo 2012 \\
\hline Calakmul-Balam Kú, Campeche & $-89.887855^{\circ}$ & $18.554372^{\circ}$ & Carrillo 2012 \\
\hline Calakmul-Balam Kú, Campeche & $-89.794702^{\circ}$ & $18.123571^{\circ}$ & Carrillo 2012 \\
\hline Calakmul-Balam Kú, Campeche & $-89.768369^{\circ}$ & $18.185786^{\circ}$ & Carrillo 2012 \\
\hline Calakmul-Balam Kú, Campeche & $-89.778974^{\circ}$ & $18.180738^{\circ}$ & Carrillo 2012 \\
\hline Calakmul-Balam Kú, Campeche & $-89.862672^{\circ}$ & $18.307547^{\circ}$ & Carrillo 2012 \\
\hline Calakmul-Balam Kú, Campeche & $-89.866658^{\circ}$ & $18.307920^{\circ}$ & Carrillo 2012 \\
\hline Calakmul-Balam Kú, Campeche & $-89.872557^{\circ}$ & $18.323994^{\circ}$ & Carrillo 2012 \\
\hline Calakmul-Balam Kú, Campeche & $-89.867077^{\circ}$ & $18.552757^{\circ}$ & Carrillo 2012 \\
\hline Calakmul-Balam Kú, Campeche & $-89.869882^{\circ}$ & $18.621391^{\circ}$ & Carrillo 2012 \\
\hline Calakmul-Balam Kú, Campeche & $-89.923296^{\circ}$ & $18.561258^{\circ}$ & Carrillo 2012 \\
\hline Calakmul-Balam Kú, Campeche & $-89.868733^{\circ}$ & $18.309508^{\circ}$ & Carrillo 2012 \\
\hline Calakmul-Balam Kú, Campeche & $-89.886102^{\circ}$ & $18.365189^{\circ}$ & Carrillo 2012 \\
\hline Calakmul-Balam Kú, Campeche & $-89.744383^{\circ}$ & $18.186563^{\circ}$ & Carrillo 2012 \\
\hline
\end{tabular}




\begin{tabular}{|c|c|c|c|}
\hline Localidad & Longitud & Latitud & Referencia \\
\hline Bacalar, Quintana Roo & $-88.295484^{\circ}$ & $18.791657^{\circ}$ & Perez-Flores 2013 \\
\hline Bacalar, Quintana Roo & $-88.275095^{\circ}$ & $18.812053^{\circ}$ & Perez-Flores 2013 \\
\hline Conhuas, Campeche & $-89.5329^{\circ}$ & $18.21388^{\circ}$ & ECOSUR 2012 \\
\hline Conhuas, Campeche & $-89.5458^{\circ}$ & $18.3220^{\circ}$ & ECOSUR 2012 \\
\hline Nuevo Becal, Campeche & $-89.1752^{\circ}$ & $18.3647^{\circ}$ & ECOSUR 2012 \\
\hline
\end{tabular}

